\title{
Prevención y manejo clínico de pre eclampsia y eclampsia: Guía de práctica clínica basada en evidencias del instituto de referencia nacional en salud materna del Perú
}

\section{Prevention and clinical management of pre-eclampsia and eclampsia: Evidence-based clinical practice guidelines from the national reference institute in maternal health of Peru}

\author{
Enrique Guevara Ríos ${ }^{1, a}$, Carlos Pérez Aliaga ${ }^{1, a}$, Walter De la Peña Meniz ${ }^{1, b}$, Oscar Limay Ríos ${ }^{1, a}$, Luis Meza \\ Santibáñez ${ }^{1, a}$, Silvia Ching Ganoza ${ }^{2, a}$, Miriam Rojas Aguedo ${ }^{1, c}$, Marlene Huayanay Bernabé, ${ }^{1, d}$, Oswaldo \\ Gonzales Carrillo ${ }^{1, a}$, Antonio Luna Figueroa ${ }^{1,4, a}$, Jenby Orihuela Salazar ${ }^{1, c}$, Nelly Angulo Rivera ${ }^{2, c}$, Karen \\ Huamán Sánchez ${ }^{3,4, e}$, Gloria Carmona Clavijo ${ }^{3,4, e}$, Catherine Bonilla Untiveros ${ }^{3,4, h}$, Celia Castillo Villacrez ${ }^{3,4, b}$, \\ Nora Reyes Puma ${ }^{3,4,}$, Patricia Caballero Ñopo ${ }^{3,4,9}$ \\ ${ }^{1}$ Instituto Nacional Materno Perinatal, Ministerio de Salud. Lima, Perú. \\ ${ }^{2}$ Red de Salud San Juan de Lurigancho, Ministerio de Salud. Lima, Perú. \\ ${ }^{3}$ Unidad de Análisis y Generación de Evidencias en Salud Pública, Instituto Nacional de Salud. Lima, Perú. \\ ${ }^{4}$ Universidad Nacional Mayor de San Marcos. Lima, Perú. \\ aédico gineco obstetra \\ ${ }^{\mathrm{b}}$ Médico intensivista \\ 'Licenciada en obstetricia \\ Médico anestesiólogo \\ ' Licenciada en enfermería, master in science \\ ${ }^{\ddagger}$ Médico cirujano, magister en salud pública \\ ${ }^{9}$ Médico infectólogo, master in science \\ h Licenciada en nutrición, magister en nutrición y alimentos
}

Correspondencia:

Gloria Carmona Clavijo

gloriacarmona.c@gmail.com

Recibido: 2 de junio 2019

Aceptado: 24 de junio 2019

Publicación en línea: 28 de junio 2019

Conflictos de interés: Los autores declaran no tener conflictos de interés

Fuente de financiamiento: Instituto Nacional de Salud e Instituto Nacional Matemo Perinatal

Contribuciones de autoría: Todos los autores participaron en el proceso de elaboración de la guía. KHS, NRP PCÑ y CPA redactaron la primera versión del artículo. Todos los autores participaron en la discusión de los estudios y la formulación de las recomendaciones de la guía en su versión extensa. Todos los autores revisaron y aprobaron la versión final del artículo.

Citar como: Guevara E, Pérez C, De la Peña W, Limay O, Meza L, Ching $S$, et al. An Fac med. Prevención y manejo clínico de pre eclampsia y eclampsia: Guía de práctica clínica basada en evidencias del instituto de referencia nacional en salud matema del Perú. 2019;80(2):243-9.DOl: https// 10.15381/anales.802.16422
An Fac med. 2019;80(2):243-9 / DOI: https://10.15381/anales.802.16422

\section{Resumen}

Introducción. En Perú, los trastomos hipertensivos del embarazo son la segunda causa de mortalidad materna. Objetivo. La presente guía de práctica clínica basada en evidencias (GPC-BE) brinda recomendaciones para la prevención y tratamiento de la pre-eclampsia y eclampsia. Métodos. Se elaboró una GPC-BE adaptada mediante un proceso sistemático, riguroso y transparente con un grupo elaborador conformado por metodólogos y profesionales de la salud expertos en el manejo de la enfermedad. La guia cuenta con dieciséis preguntas clínicas y dieciséis recomendaciones. Se realizó la búsqueda y preselección de GPC, las mismas que fueron evaluadas mediante el instrumento "Appraisal of Guidelines for Research and Evaluation II" (AGREEII), para su adaptación. La búsqueda sistemática de evidencias para responder las preguntas clínicas se realizó en múltiples bases de datos: MEDLINE/PubMed, EMBASE/Ovid, Cochrane Library, LILACS y SciELO. Las evidencias fueron seleccionadas y analizadas críticamente por pares clínicos y metodológicos; las recomendaciones fueron elaboradas mediante la metodología Grading of Recommendations Assessment, Development, and Evaluation (GRADE). Resultados. Con la participación de un panel de expertos clínicos del Instituto Nacional Matemo Perinatal, redes de salud y otras instituciones de referencia, se elaboraron 16 recomendaciones dirigidas a la prevención y tratamiento de mujeres gestantes en riesgo de pre-eclampsia, gestantes con pre-eclampsia, eclampsia o enfermedad hipertensiva. Conclusiones. Este artículo resume la guía de práctica clínica basada en evidencias para la prevención y tratamiento de pre-eclampsia y eclampsia en gestantes en el instituto de referencia nacional en salud matema y neonatal del Perú.

Palabras clave: Preeclampsia; Eclampsia; Hipertensión Inducida en el Embarazo; Guía de Práctica Clínica; Medicina Basada en Evidencias; Enfoque GRADE

Abstract

Introduction. In Peru, hypertensive disorders of pregnancy are the second cause of maternal mortality. Objective. This evidence-based clinical practice guideline (EB-CPG) provides recommendations for the prevention and treatment of preeclampsia and eclampsia. Methods. An adapted EB-CPG was elaborated through a systematic, rigorous and transparent process with a group consisting of methodologists and health professionals who are experts in the management of the disease. The guide has sixteen clinical questions and sixteen recommendations. The search and pre-selection of EB-CPGs were performed, which were evaluated through the "Appraisal of Guidelines for Research and Evaluation II" tool (AGREE-II), for its adaptation. The systematic search for evidence to answer the 16 clinical questions were made in multiple databases: MEDLINE / PubMed, EMBASE / Ovid, Cochrane Library, LLACS and SciELO. Evidences were selected and analyzed critically by clinicians and methodologists in pairs, recommendations were elaborated through the Grading of Recommendations Assessment, Development, and Evaluation methodology (GRADE). Results. With the participation of a panel of clinical experts from the National Perinatal Maternal Institute, representatives from health care networks and other reference institutions, 16 recommendations were established for the prevention and treatment of pregnant women at risk of preeclampsia, for pregnant women with pre-eclampsia, eclampsia or hypertensive disease. Conclusions. This article summarizes the evidence-based clinical practice guidelines for the prevention and treatment of preeclampsia and eclampsia in peruvian pregnant women for the national reference institute for maternal and neonatal health of Peru.

Keywords: Pre-eclampsia; Eclampsia; Hypertension, Pregnancy-Induced; Clinical Practice Guidelines; EvidenceBased Medicine; GRADE Approach 


\section{INTRODUCCIÓN}

La enfermedad hipertensiva del embarazo causa el $21 \%$ de muertes maternas en el país, representando el segundo lugar. En los años 2015, 2016, 2017 y 2018 se notificaron en total 415 328, 377 y 362 muertes maternas respectivamente, siendo que cada año la mayoría de mujeres fallecidas se encuentran entre los 20 y 35 años de edad ${ }^{1}$.

El manejo clínico de pre-eclampsia y eclampsia ha sido siempre abordado en normas técnicas y directivas del Ministerio de Salud del Perú. Estas directivas tradicionalmente han priorizado la administración del tratamiento sólo en servicios de salud del tercer nivel de atención, y la aplicación de criterios para el traslado de las pacientes desde los centros de salud materno infantil del primer nivel. Los reportes estadísticos evidenciaron que este tipo de recomendaciones generaron la derivación de gestantes con preeclampsia o eclampsia con criterios de severidad que fallecieron en el Instituto Nacional Materno Perinatal (INMP), limitando la posibilidad de detección y manejo temprano. Asimismo, respecto a las alternativas de tratamiento, ha existido variabilidad en la elección de las opciones farmacológicas, por limitaciones en el uso de evidencia para tomar decisiones clínicas.

En este contexto, siguiendo las directrices metodológicas aprobadas en el 2015 para el desarrollo de guías de práctica clínica basadas en evidencia (GPC BE)², las autoridades y profesionales del INMP -institución de referencia nacional en salud materna y neonatal-, en colaboración con el Instituto Nacional de Salud (INS), a través de la Unidad de Análisis y Generación de Evidencias en Salud Pública (UNAGESP), elaboraron la "Guía de Práctica Clínica para la prevención y manejo de la Pre-eclampsia y Eclampsia"3. Esta guía brinda recomendaciones basadas en evidencia para la atención de las gestantes en riesgo de pre-eclampsia, gestantes con pre-eclampsia o eclampsia, o con enfermedad hipertensiva. Tiene como principales aportes las recomendaciones dirigidas al primer nivel de atención para reducir el riesgo de pre-eclampsia o para iniciar la administración de sulfato de magnesio antes de trasladar a la gestante. Del mismo modo, son un aporte innovador las recomendaciones para disminuir la variabilidad en el tratamiento de pre-eclampsia y eclampsia en el tercer nivel o para definir la decisión por el manejo expectante versus la culminación del embarazo según la edad gestacional de la paciente. Todas las recomendaciones han sido generadas por un grupo elaborador multidisciplinario de profesionales del INMP y los diferentes niveles de atención quienes, a través de un proceso estratégico de transferencia tecnológica, incorporaron competencias metodológicas para el desarrollo de esta y otras futuras GPC BE.

\section{METODOLOGÍA}

Se realizó un proceso metodológico de adaptación de GPC-BE de manera rigurosa, sistemática y transparente usando el enfoque GRADE. La versión extensa se encuentra disponible en: https://web. ins.gob.pe/sites/default/files/Archivos/ cnsp/unagesp/Guia_de_Practica_Clinica_para_la_prevencion_y_manejo_de_ Preeclampsia_y_Eclampsia_Version_extensa_v3.pdf

Conformación de los grupos y panel de expertos: se conformó un grupo metodológico (GM) integrado por profesionales de la UNAGESP que condujeron el proceso de transferencia tecnológica y asesoramiento técnico a los profesionales médicos gineco-obstetras, intensivistas, anestesiólogos y otras profesiones de salud, pertenecientes al INMP, instituciones del primer y segundo nivel de atención y la Sociedad Peruana de Ginecología y Obstetricia; todos con amplia experiencia en el manejo clínico de preeclampsia y eclampsia. El objetivo de la guía fue brindar recomendaciones basadas en evidencia científica con énfasis en la prevención y el manejo temprano de la pre-eclampsia y eclampsia.

Selección de guías internacionales y formulación de preguntas PICO: el proceso de identificación de guías internacionales de calidad se realizó por los asesores regionales en guías de práctica clínica de la Organización Panamericana de la Salud (OPS), el equipo metodoló- gico de la Universidad McMaster (Canadá) y el INS. Se identificaron y seleccionaron dos GPC-BE sobre pre-eclampsia y eclampsia desarrolladas por Arabia Saudita ${ }^{4,5}$. Para determinar su nivel de calidad fueron evaluadas utilizando el instrumento AGREE $\|^{6}$, resultando ambas guías de buena calidad metodológica. El grupo elaborador (GE) formuló preguntas clínicas en formato PICO (population, intervention, comparison y outcome, por sus siglas en inglés) y las comparó con las preguntas clínicas incluidas en las guías de Arabia Saudita. Posteriormente, analizó la relevancia de cada pregunta para el contexto local y convocó a un grupo ampliado de profesionales denominado "panel de expertos", con el cual, a modo de consenso desarrollaron un proceso de priorización y validación final de las preguntas PICO.

Revisión y síntesis de evidencia: se replicaron y actualizaron las estrategias de búsqueda sistemática de las guías originales en las bases de datos Cochrane Library, MEDLINE, LILACS y SciELO; estas dos últimas a fin de encontrar evidencias regionales y nacionales sobre valores y preferencias de los pacientes.

Gradación del cuerpo de la evidencia: se seleccionó la evidencia resultante de la búsqueda por título y resumen por el GE, luego se revisaron a texto completo los artículos seleccionados y las discrepancias entre revisores fueron resueltas por consenso. La calificación del cuerpo de la evidencia se realizó utilizando la metodología GRADE, según se describe en la tabla 1.

Formulación de recomendaciones y diálogo deliberativo: para la formulación de recomendaciones se consideró la calidad de la evidencia, el balance riesgo-beneficio, la factibilidad de la intervención y los costos implicados. Para incorporar la perspectiva de los pacientes, se realizó una búsqueda de evidencia cualitativa sobre sus valores y preferencias. El GE con el panel de expertos discutieron estos aspectos y plantearon las recomendaciones finales durante el diálogo deliberativo, cuya interpretación se muestra en la tabla 2, y utilizando la metodología GRADE. 
Tabla 1. Interpretación de niveles de evidencia según la metodología GRADE.

\begin{tabular}{ll}
$\begin{array}{l}\text { Nivel de } \\
\text { evidencia } \\
\text { GRADE }\end{array}$ & \multicolumn{1}{c}{ Características } \\
Alta & $\begin{array}{l}\text { Existe muy poca probabilidad } \\
\text { que nuevos estudios cambien } \\
\text { la certeza de los resultados. }\end{array}$ \\
\hline Moderada & $\begin{array}{l}\text { Probablemente puedan } \\
\text { existir nuevos estudios que } \\
\text { influyan en la confianza de } \\
\text { los resultados estimados y } \\
\text { puedan modificarlo. }\end{array}$ \\
\hline Baja & $\begin{array}{l}\text { Es muy probable que nuevos } \\
\text { estudios influyan en la } \\
\text { confianza de los resultados } \\
\text { estimados y los modifiquen. }\end{array}$ \\
\hline Muy baja & $\begin{array}{l}\text { Cualquier resultado estimado } \\
\text { es muy incierto. }\end{array}$ \\
\hline
\end{tabular}

Desarrollo de recomendaciones de la guía

La guía cuenta con 16 preguntas PICO (mostradas en las tablas 3 a 6 ) y recomendaciones orientadas a la prevención y tratamiento (tabla 7) de la pre-eclampsia y eclampsia, lo que incluye el manejo farmacológico y la decisión clínica intervencionista versus expectante respecto a la continuación del embarazo.

A continuación se describe el proceso de desarrollo basado en la metodología GRADE:

Tabla 2. Interpretación de la fuerza de recomendaciones según la metodología GRADE.

\begin{tabular}{|c|c|}
\hline $\begin{array}{l}\text { Fuerza de la } \\
\text { recomendación }\end{array}$ & Significado \\
\hline Fuerte a favor & $\begin{array}{l}\text { Los efectos deseables superan } \\
\text { los efectos indeseables. } \\
\text { Se recomienda hacerlo }\end{array}$ \\
\hline $\begin{array}{l}\text { Condicional a } \\
\text { favor }\end{array}$ & $\begin{array}{l}\text { Los efectos deseables } \\
\text { probablemente superan los } \\
\text { efectos indeseables. } \\
\text { Se sugiere hacerlo }\end{array}$ \\
\hline $\begin{array}{l}\text { Condicional en } \\
\text { contra }\end{array}$ & $\begin{array}{l}\text { Los efectos indeseables } \\
\text { probablemente superan los } \\
\text { efectos deseables. } \\
\text { Se sugiere no hacerlo }\end{array}$ \\
\hline $\begin{array}{l}\text { Fuerte en } \\
\text { contra }\end{array}$ & $\begin{array}{l}\text { Los efectos indeseables } \\
\text { superan los efectos deseables. } \\
\text { Se recomienda no hacerlo }\end{array}$ \\
\hline
\end{tabular}

Tabla 3. Lista de Preguntas PICO sobre prevención de pre-eclampsia.

Tema $\mathbf{N}^{\circ} \quad$ Pregunta

¿En las gestantes, el utilizar suplementación con calcio en

PICO 1 comparación con no utilizarla, actúa favorablemente en la reducción del riesgo de pre-eclampsia?

¿En las gestantes, el utilizar suplementación con vitamina

PICO 2 D en comparación con no utilizarla, actúa favorablemente en la reducción del riesgo de pre-eclampsia?

Prevención de pre eclampsia ¿En las gestantes con riesgo de desarrollar preeclampsia, utilizar (o administrar) ácido acetilsalicílico en comparación con la no utilización, actúa favorablemente en la reducción del riesgo de pre-eclampsia?

PICO 3 eclampsia, utilizar (o administrar) ácido acetilsalicílico en
comparación con la no utilización, actúa favorablemente
en la reducción del riesgo de pre-eclampsia?

¿En las gestantes con hipertensión arterial, practicar

PICO 4 reposo en cama en comparación con actividades no restringidas actúa favorablemente en la reducción del riesgo de pre-eclampsia?

\section{PREVENCIÓN DE LA PRE ECLAMPSIA}

Pregunta 1. ¿En las gestantes, el utilizar suplementación con calcio en comparación con no utilizarla, actúa favorablemente en la reducción del riesgo de pre-eclampsia?

\section{De la evidencia científica a la práctica} clínica

La suplementación con calcio redujo la hipertensión, siendo el beneficio mayor

Tabla 4. Lista de Preguntas PICO sobre tratamiento de pre-eclampsia.

\section{Tema} go. Este suplemento es financiado por el Seguro Integral de Salud y según reportes de International Network of Agencies for Health Technology Assessment (INA$\mathrm{HTA})^{7}$, la suplementación de calcio es una intervención costo efectiva ${ }^{8}$.

Pregunta 2. ¿En las gestantes, el utilizar suplementación con vitamina D en comparación con no utilizarla, actúa favorablemente en la reducción del riesgo de pre-eclampsia?

\section{$\mathbf{N}^{\circ} \quad$ Pregunta \\ $\mathbf{N}^{\circ} \quad$ Pregunta}

\begin{tabular}{cl} 
PICO 5 & $\begin{array}{l}\text { ¿Debería usarse sulfato de magnesio o fenitoína en } \\
\text { gestantes con pre-eclampsia, a fin de limitar su evolución } \\
\text { hacia la eclampsia? }\end{array}$ \\
\hline PICO 6 & $\begin{array}{l}\text { ¿Debería usarse o no usarse sulfato de magnesio en } \\
\text { gestantes con pre eclampsia con criterios de severidad? }\end{array}$ \\
\hline PICO 7 & $\begin{array}{l}\text { ¿Debería usarse sulfato de magnesio o diazepam en } \\
\text { gestantes con pre-eclampsia con criterios de severidad? }\end{array}$ \\
\hline PICO 8 & $\begin{array}{l}\text { ¿Se debería culminar la gestación o mantenerse una } \\
\text { conducta expectante en mujeres con pre-eclampsia con } \\
\text { criterios de severidad y con menos de 24 semanas de edad } \\
\text { gestacional? }\end{array}$ \\
\hline PICO 9 & $\begin{array}{l}\text { ¿Se debería culminar la gestación o mantenerse una } \\
\text { conderios de severidad y entre las 24 a } 33 \text { semanas más } 6 \\
\text { días de edad gestacional? }\end{array}$
\end{tabular}

Tratamiento de pre eclampsia días de edad gestacional?

PICO 10

¿Se debería culminar la gestación o mantenerse una conducta expectante en mujeres con pre-eclampsia con criterios de severidad y entre las 34 a 36 semanas más 6 días de edad gestacional?

¿Se debería culminar la gestación o mantenerse una conducta

PICO 11 expectante en mujeres con pre-eclampsia con criterios de severidad después de las 37 semanas de edad gestacional? 
Tabla 5. Lista de Preguntas PICO sobre tratamiento de eclampsia.

\begin{tabular}{lll} 
Tema & \multicolumn{1}{c}{$\mathbf{N}^{\circ}$} & \multicolumn{1}{c}{ Pregunta } \\
\cline { 2 - 2 } $\begin{array}{l}\text { Tratamiento de } \\
\text { eclampsia }\end{array}$ & PICO 12 & $\begin{array}{l}\text { ¿Debería usarse sulfato de magnesio o fenitoína en gestantes } \\
\text { con eclampsia? }\end{array}$ \\
\cline { 2 - 3 } & PICO 13 & $\begin{array}{l}\text { ¿Debería usarse sulfato de magnesio o diazepam en gestantes } \\
\text { con eclampsia? }\end{array}$ \\
\hline
\end{tabular}

\section{De la evidencia científica a la práctica clínica}

La administración de vitamina $\mathrm{D}$ podría reducir el riesgo de pre-eclampsia, pero cuando se administra asociada con el calcio se incrementa el riesgo de parto prematuro, por ello se sostiene la necesidad de estudios de más alta calidad que puedan demostrar la seguridad y eficacia de su uso ${ }^{9,10}$.

Pregunta 3. ¿En las gestantes con riesgo de desarrollar pre-eclampsia, utilizar (o administrar) ácido acetilsalicílico en comparación con la no utilización, actúa favorablemente en la reducción del riesgo de pre-eclampsia?

\section{De la evidencia científica a la práctica clínica}

El impacto de esta intervención es aceptable y factible considerando que el ácido acetilsalicílico está incluido en el Petitorio Nacional Único de Medicamentos (PNUME) $)^{7,11,12}$.

Pregunta 4. ¿En las gestantes con hipertensión arterial, practicar reposo en cama en comparación con actividades no restringidas actúa favorablemente en la reducción del riesgo de pre-eclampsia?

\section{De la evidencia científica a la práctica clínica}

No se ha logrado identificar evidencia suficiente que sustente los beneficios o

Tabla 6. Lista de Preguntas PICO sobre hipertensión y Síndrome de HELLP.

\section{Tema} $\mathbf{N}^{\circ}$ Pregunta

PICO 14 ¿Debería usarse o no usarse tratamiento oral antihipertensivo para la prevención de la hipertensión en el post parto?

\begin{tabular}{lll} 
Hipertensión & \multicolumn{2}{c}{$\begin{array}{l}\text { arterial y } \\
\text { sindrome de } \\
\text { hellp }\end{array}$} \\
\cline { 2 - 3 } & PICO 15 & $\begin{array}{l}\text { ¿En las gestantes con Síndrome de HELLP, el uso de esteroides } \\
\text { en comparación con la no utilización, actúa favorablemente en } \\
\text { la evolución de la enfermedad? }\end{array}$ \\
\cline { 2 - 3 } & PICO 16 & $\begin{array}{l}\text { ¿Debería usarse o no usarse antihipertensivos en gestantes con } \\
\text { hipertensión leve? }\end{array}$ \\
\hline
\end{tabular}

de pre-eclampsia, hubo reducción no significativa de la mortalidad materna y se redujo el riesgo de desprendimiento prematuro de placenta ${ }^{16}$. Los efectos colaterales (disminución de frecuencia respiratoria y de frecuencia cardiaca) se encontraron en el $25 \%$ de los casos, siendo sin embargo menores a los que se presentan con otros anticonvulsivantes ${ }^{17-20}$.

El panel no recomienda el uso de otros anticonvulsivantes como fenobarbital o diazepam, por el contrario se propicia el uso del sulfato de magnesio desde el el traslado al mayor nivel de complejidad. En los anexos de la versión extensa de esta guía se pueden encontrar las pautas de administración del sulfato de magnesio del INMP3.

Pregunta 7. ¿Debería usarse sulfato de magnesio o diazepam en gestantes con pre eclampsia con criterios de severidad? de magnesio o fenitoína en gestantes con pre eclampsia, a fin de limitar su evolución hacia la eclampsia?

\section{De la evidencia científica a la práctica clínica}

El sulfato de magnesio es bien tolerado con bajos efectos colaterales ${ }^{14}$. Por su bajo costo $^{15}$, es accesible en todos los niveles de atención, para ser administrado antes del traslado de la gestante al tercer nivel. Su uso contribuiría a la equidad en la atención. El panel de la guía no recomienda el uso de fenitoína en ningún nivel de atención.

Pregunta 6. ¿Debería usarse o no usarse sulfato de magnesio en gestantes con pre eclampsia con criterios de severidad?

\section{De la evidencia científica a la práctica clínica}

Con al uso del sulfato de magnesio disminuyó en más de la mitad el riesgo

\section{De la evidencia científica a la práctica clínica}

El sulfato de magnesio ha demostrado ser más efectivo clínicamente que cualquier otro anticonvulsivante, y su nivel de toxicidad en relación a depresión ventilatoria en comparación con diazepam es bajo ${ }^{14,17}$. El panel considera que el impacto de la intervención es aceptable y factible de incrementar la equidad en salud $^{18-20}$.

Pregunta 8. ¿Se debería culminar la gestación o mantenerse una conducta expectante en mujeres con pre eclampsia con criterios de severidad y con menos de 24 semanas de edad gestacional?

Se considera culminación de la gestaprimer nivel de atención antes de hacer

\section{De la evidencia científica a la práctica clínica} ción como el tratamiento intervencionista basado en la inducción del trabajo de parto o la cesárea; y se considera manejo expectante la decisión de continuar con el embarazo con monitoreo materno y fetal estricto ${ }^{11,18,21}$. El panel de la guía consideró que mediante la conducta intervencionista la posibilidad de supervivencia perinatal de un feto de igual o menor a 24 semanas es mínima; por el contrario, el beneficio a la madre es muy alto.

Pregunta 9. ¿Se debería culminar la gestación o mantenerse una conducta 
Tabla 7. Recomendaciones de la guía de práctica clínica basada en evidencias para el manejo de la pre-eclampsia y eclampsia.

Tema $\quad \mathbf{N}^{\circ} \quad$ Recomendación basada en evidencias

PICO 1 Se recomienda usar suplementos de calcio (1g por día) para la reducción del riesgo de pre-eclampsia.

PICO 1 Recomendación fuerte a favor de la intervención basada en evidencia de moderada calidad.

PICO 2 No se recomienda usar suplementación con vitamina D para la reducción del riesgo de pre-eclampsia.

Recomendación condicional en contra de la intervención basada en evidencia de muy baja calidad.

Prevención de pre eclampsia
Se recomienda usar ácido acetilsalicílico en gestantes con riesgo de desarrollar pre-eclampsia. Recomendación fuerte a favor de la intervención basada en evidencia de baja calidad.

PICO 3 Consideraciones: El panel sugiere tomar en cuenta la edad gestacional, considerando que hay evidencia que demuestra que el ácido acetilsalicílico no tiene efecto si se inicia después de las 16 semanas. Por lo tanto, se sugiere iniciar su uso antes de las 16 hasta las 34 semanas de gestación en mujeres en alto riesgo. Además, las pacientes con riesgo de desarrollar pre-eclampsia deberían seguir su control prenatal en establecimientos de mayor complejidad.

Se sugiere el reposo en cama en gestantes con hipertensión.

PICO 4 Recomendación condicional a favor de la intervención basada en evidencia de baja calidad.

Consideraciones: Se debe tomar en cuenta los valores y preferencias de la gestante, además de especificar las características del reposo en cama (absoluto, relativo, etc.).

PICO 5 Se recomienda el sulfato de magnesio en lugar de fenitoína en gestantes con pre-eclampsia con signos de severidad. Recomendación fuerte a favor del uso de sulfato de magnesio basada en evidencia de muy baja calidad.

Se recomienda el sulfato de magnesio en gestantes con pre-eclampsia con criterios de severidad.

Recomendación fuerte a favor de la intervención basada en evidencia de moderada calidad.

Consideraciones: Se requiere contar con protocolos de atención que especifiquen dosis y vía de administración según el nivel de atención del establecimiento de salud. La versión extensa incluye pautas para la referencia de las gestantes. Se considera preeclampsia con criterios de severidad si presenta alguno de los signos establecidos por la "American College of Obstetricians and Gynecologists" (ACOG):

PICO 6 - PA sistólica mayor o igual a $160 \mathrm{mmHg}$ o PA diastólica mayor o igual a $110 \mathrm{mmHg}$

- trombocitopenia mayor a 1000 000/mL

compromiso de función hepática: elevación de transaminasas o dolor en cuadrante superior derecho o epigastrio creatinina mayor de $1,1 \mathrm{mg} / \mathrm{dL}$

trastornos cerebrales o visuales

edema pulmonar o cianosis insuficiencia renal progresiva

Tratamiento de pre-eclampsia

PICO 7 Se recomienda sulfato de magnesio en lugar de diazepam en gestantes con pre-eclampsia severa.

PICO 7 Recomendación fuerte a favor del uso de sulfato de magnesio basada en evidencia de baja calidad. Se sugiere culminar el embarazo en gestantes con pre-eclampsia con criterios de severidad con menos de 24 semanas de edad gestacional.

PICO 8 Recomendación condicional a favor de la culminación del embarazo basada en evidencia de baja calidad. Consideraciones: Esta recomendación prioriza la salud de la madre la cual está alineada a los valores y preferencias de la mayoría de las pacientes.

Se recomienda la conducta expectante en gestantes con preeclampsia con criterios de severidad entre las 24 a 33 semanas y 6 días de edad gestacional; siempre que no exista una hipertensión arterial incontrolada, compromiso de la funcionalidad de algún

PICO 9 órgano blanco o distrés fetal demostrado mediante monitoreo.

Consideraciones: La conducta expectante significa continuar el embarazo bajo monitoreo materno fetal estricto (en unidades de cuidados intensivos, cuidados intermedios o unidades de alto riesgo obstétrico).

PICO 10 Se sugiere culminar el embarazo en gestantes con preeclampsia con criterios de severidad entre las 34 a 36 semanas y 6 días de edad gestacional. Recomendación fuerte a favor de la culminación del embarazo basada en evidencia de baja calidad.

Se recomienda culminar el embarazo en gestantes con pre-eclampsia con criterios de severidad con más de 37 semanas de edad

PICO 11 gestacional.

Recomendación fuerte a favor de la culminación del embarazo basada en evidencia de baja calidad.

Tratamiento de Se recomienda usar sulfato de magnesio en lugar de fenitoína en gestantes con eclampsia.

PICO 12 Recomendación fuerte a favor del uso de sulfato de magnesio basada en evidencia de baja calidad.

eclampsia

PICO 13 Se sugiere usar sulfato de magnesio en lugar de diazepam en gestantes con eclampsia.

Recomendación fuerte a favor del uso de sulfato de magnesio basada en evidencia de baja calidad.

El panel de esta GPC no puede emitir una recomendación a favor o en contra de usar o no usar el tratamiento oral anti-hipertensivo en el post-parto en pacientes que han tenido hipertensión durante el embarazo para prevención de la hipertensión. Los hallazgos de

PICO 14 la evidencia encontrada fueron no concluyentes

Consideración: el tratamiento antihipertensivo será para el manejo de hipertensión en el post parto, pero no será con fines de "prevención".

arterial y

sindrome de

hellp

PICO 15 Se sugiere no usar esteroides en el gestantes con Síndrome de HELLP.

Recomendación condicional en contra de la intervención basada en evidencia de muy baja calidad.

PICO 16 Se recomienda no usar antihipertensivos en gestantes con hipertensión leve.

PICO 16 Recomendación condicional en contra de la intervención basada en evidencia de muy baja calidad. 
expectante en mujeres con pre-eclampsia con criterios de severidad y entre las 24 a 33 semanas y 6 días de edad gestacional?

\section{De la evidencia científica a la práctica clínica}

En las gestantes con estas características la letalidad es alta, pero la posibilidad de supervivencia perinatal aumenta conforme avanza la gestación, siendo necesario el monitoreo. El manejo expectante es posible por las mejores condiciones para el nacimiento y atención de un prematuro. El riesgo de esta decisión es la evolución desfavorable hacia eclampsia y si durante el manejo expectante se aprecia compromiso de órgano blanco, es necesario culminar el embarazo.

Pregunta 10. ¿Se debería culminar la gestación o mantenerse una conducta expectante en mujeres con pre-eclampsia con criterios de severidad y entre las 34 a 36 semanas y 6 días de edad gestacional?

\section{De la evidencia científica a la práctica} clínica

Se recomienda la culminación del embarazo en este grupo porque el beneficio para los recién nacidos con el tratamiento expectante es incierto y existe riesgo de progresión de la pre-eclampsia ${ }^{18,21-23}$. Este manejo evitará la progresión de la hipertensión y sus complicaciones (eclampsia, síndrome de HELLP, desprendimiento prematuro de placenta, ruptura hepática).

Pregunta 11. ¿Se debería culminar la gestación o mantenerse una conducta expectante en mujeres con pre-eclampsia con criterios de severidad después de las 37 semanas de edad gestacional?

\section{De la evidencia científica a la práctica clínica}

El panel consideró la culminación del embarazo como la mejor alternativa en gestantes a término. El beneficio es la no exposición a la progresión de la enfermedad para ambos ${ }^{23}$.

\section{TRATAMIENTO DE LA ECLAMPSIA}

Pregunta 12. ¿¿Debería usarse sulfato de magnesio o fenitoína en gestantes con eclampsia?
De la evidencia científica a la práctica clínica

El sulfato de magnesio se asocia con una reducción sustancial de la recurrencia de las convulsiones, en comparación con fenitoína. Su nivel de toxicidad en relación a depresión ventilatoria es bajo. Asimismo, es un medicamento de bajo costo, que lo hace accesible $e^{14}$.

Pregunta 13. ¿Debería usarse sulfato de magnesio o diazepam en gestantes con eclampsia?

\section{De la evidencia científica a la práctica clínica}

El sulfato de magnesio se asocia con reducción de la mortalidad materna y recurrencia de convulsiones en comparación con diazepam ${ }^{11}$.

\section{HIPERTENSIÓN ARTERIAL Y SINDROME DE HELLP}

Pregunta 14. ¿Debería usarse o no usarse tratamiento oral antihipertensivo, para la prevención de la hipertensión en el post parto?

De la evidencia científica a la práctica clínica

Se concluye que se requiere mayor evidencia antes de recomendar esta práctica $^{23,24}$.

Pregunta 15. ¿En las gestantes con Síndrome de HELLP, el uso de esteroides en comparación con la no utilización, actúa favorablemente en la evolución de la enfermedad?

\section{De la evidencia científica a la práctica} clínica

De acuerdo a los estudios clínicos revisados, no existe beneficio con el uso de esteroides en el síndrome de HELLP ${ }^{25}$.

Pregunta 16. ¿Debería usarse o no usarse antihipertensivos en gestantes con hipertensión leve?

\section{De la evidencia científica a la práctica clínica}

Aún no existe evidencia suficiente que sustente los beneficios o daños de esta intervención. Se deben tomar en cuenta los desenlaces que pueden presentarse en el feto ${ }^{16,19,26}$.

\section{RECOMENDACIONES PARA LA INVESTIGACIÓN}

Durante el proceso de dialogo deliberativo con aplicación del sistema GRADE, se identificaron las siguientes necesidades de investigación:

a. Evaluación del riesgo de hipertensión y otras complicaciones en gestantes que han presentado hipertensión durante el embarazo.

b. Uso de sulfato de magnesio en pacientes con pre eclampsia sin signos de severidad.

c. Beneficios de la administración de sulfato de magnesio vía intramuscular versus intravenosa.

d. Preferencias y valores de las gestantes con pre eclampsia en relación al reposo en cama versus actividades no restringidas.

\section{AGRADECIMIENTOS}

Al Dr. Carlos Cuello, profesor de la Universidad Mac Master, Canadá, por su asesoramiento metodológico. A los doctores José Pacheco Romero y Pedro Saona Ugarte por aceptar ser los revisores externos nacionales de la guía. Y al Dr. Ludovic Reveiz Herault, médico epidemiólogo de la Organización Panamericana de la Salud Sede Washington, por su asesoramiento como revisor metodológico internacional.

\section{REFERENCIAS BIBLIOGRÁFICAS}

1. Dirección General de Epidemiologia, Ministerio de Salud del Perú [Internet]. Boletín Epidemiológico (Lima - Perú), volumen 24 - semana epidemiológica No 4, 2016 [Fecha de acceso: 23 de mayo 2019]. Disponible en: http://www.dge.gob.pe/portal/docs/ vigilancia/boletines/2016/04.pdf

2. Dirección General de Salud de las Personas, Ministerio de Salud, Perú [Internet]. Documento Técnico: "Metodologia para la elaboración de Guias de Práctica Clínica". RM N 414-2015/MINSA [Fecha de acceso: 23 de mayo 2019]. Disponible en: http:// bvs.minsa.gob.pe/local/MINSA/3301.pdf

3. Ministerio de Salud del Perú [Internet]. Instituto Nacional Materno Perinatal, Instituto Nacional de Salud. Guía de práctica clínica para la prevención y manejo de Preeclampsia y eclampsia -versión extensa-, 2017 [Fecha de acceso: 23 de mayo 2019]. Disponible en: http://bvs.minsa.gob.pe/local/ MINSA/4220.pdf 
4. Saudi Center for Evidence Based Health Care [Internet]. Clinical Practice Guideline on Managements of Eclampsia [Fecha de acceso: 17 de mayo 2019]. Disponible en: http://www.moh.gov.sa/endepts/ Proofs/Pages/Guidelines.aspx

5. Saudi Center for Evidence Based Health Care [Internet]. Clinical Practice Guideline on Managements of Pre-Eclampsia [Fecha de acceso: $17 \mathrm{de}$ mayo 2019]. Disponible en: http://www.moh.gov.sa/ endepts/Proofs/Pages/Guidelines.aspx

6. Consorcio AGREE, GuiaSalud, España. Instrumento AGREE II: Instrumento para la evaluación de guias de práctica clínica-versión español- [Internet]. Disponible en: http://www.guiasalud.es/contenidos/ documentos/Guias_Practica_Clinica/SpanishAGREE-II.pdf

7. Meads CA, Cnossen JS, Meher S, Juarez-Garcia A ter Riet G, Duley L, et al. Methods of prediction and prevention of pre-eclampsia: systematic reviews of accuracy and effectiveness literature with economic modelling. Health Technol Assess Winch Engl. 2008;12(6):iii-iv, 1-270.

8. Chicaíza-Becerra LA, Garcia-Molina M, OviedoAriza SP, Urrego-Novoa JR, Rincón-Rodríguez CJ, Rubio-Romero JA, et al. Costo efectividad del suplemento de calcio para reducir la mortalidad materna asociada a preeclampsia en Colombia. Rev Salud Pública. 2016;18:300-10. DOI: http://dx.doi. org/10.15446/rsap.v18n2.48776

9. De-Regil LM, Palacios C, Ansary A, Kulier R, PenaRosas JP. Vitamin D supplementation for women during pregnancy. Cochrane Database Syst Rev. 2012;(2):CD008873. DOI: 10.1002/14651858. CD008873.pub2

10. Ben W J Mol, Claire T Roberts, Shakila Thangaratiam, Laura A Magee, Christianne J M de Groot, Justus Hofmeyr. Pre-eclampsia. Lancet. 2016;387(10022):999-1011. DOI: https://doi. org/10.1016/S0140-6736(15)00070-7

11. Duley L, Henderson-Smart DJ, Meher S, King JF. Antiplatelet agents for preventing pre-eclampsia and its complications. Cochrane Database Syst Rev. 2007;(2):CD004659. DOI: 10.1002/14651858. CD004659.pub2

12. Askie L, Henderson-Smart D, Ko H. Restricted versus liberal oxygen exposure for preventing morbidity and mortality in preterm or low birth weight infants. Cochrane Database Syst Rev. 2009(1):CD001077. DOI: 10.1002/14651858.CD001077.pub2

13. Meher S, Abalos E, Carroli G. Bed rest with or without hospitalisation for hypertension during pregnancy. Cochrane Database Syst Rev. 2005;(4):CD003514. DOI: 10.1002/14651858.CD003514.pub2

14. Duley L, Gulmezoglu AM, Henderson-Smart DJ, Chou D. Magnesium sulfate and other anticonvulsants for women with preeclampsia. Cochrane Database of Systematic Reviews. 2010;(11):CD000025 DOI: 10.1002/14651858.CD000025.pub2.

15. Zakiyah N, Postma MJ, Baker PN, van Asselt AD IMPROvED Consortium. Pre-eclampsia Diagnosis and Treatment Options: A Review of Published Economic Assessments. PharmacoEconomics. 2015;33(10):1069-82. DOI: 10.1007/s40273-0150291-x

16. Abalos E, Duley L, Steyn DW, Henderson-Smart DJ. Antihypertensive drug therapy for mild to moderate hypertension during pregnancy. Cochrane Database Syst Rev. 2007;(1):CD002252. DOI: 10.1002/14651858.CD002252.pub2

17. American Collegue of Obstetricians and Gynecologist [Internet]. Task Force on Hipertension in Pregnancy. [Fecha de acceso: 13 de marzo de 2017]. Disponible en: http://www.acog.org/ Resources-And-Publications/Task-Force- andWork-Group-Reports/Hypertension-in-Pregnancy

18. Souza NL, Araujo ACPF, Costa ICC. Significados atribuidos por puérperas às síndromes hipertensivas da gravidez e nascimento prematuro. Rev Esc Enferm USP. 2011;45(6):1285-92.

19. Dirección General de Medicamentos, Insumos y Drogas, Ministerio de Salud del Perú [Internet]. Petitorio Nacional Único de Medicamentos Esenciales,
2015 [Fecha de acceso: 22 de abril 2019]. Disponible en: http://www.digemid.minsa.gob.pe/UpLoad/ UpLoaded/PDF/Normatividad/2015/RM_399-2015. pdf

20. Simon J, Gray A, Duley L, Magpie Trial Collaborative Group. Cost-effectiveness of prophylactic magnesium sulphate for 9996 women with pre-eclampsia from 33 countries: economic evaluation of the Magpie Trial. BJOG. 2006;113(2):144-51. DOI: 10.1111/j.1471-0528.2005.00785.x

21. Churchill D, Duley L, Thornton JG, Jones L. Interventionist versus expectant care for severe preeclampsia between 24 and 34 weeks' gestation. Cochrane Database Syst Rev. 2013;(7):CD003106. DOI: 10.1002/14651858.CD003106.pub2

22. National Collaborating Centre for Women's and Children's Health (UK). Hypertension in Pregnancy: The Management of Hypertensive Disorders During Pregnancy. London: RCOG Press; 2010.

23. Koopmans CM, Bijlenga D, Groen H, Vijgen SM, Aarnoudse JG, Bekedam DJ, et al. Induction of labour versus expectant monitoring for gestational hypertension or mild pre-eclampsia after 36 weeks' gestation (HYPITAT): a multicentre, open-label randomised controlled trial. Lancet. 2009;374(9694):979-88. DOI: 10.1016/S0140-6736(09)60736-4

24. Magee L, von Dadelszen P. Prevention and treatment of postpartum hypertension. Cochrane Database Syst Rev. 2013;(4):CD004351. DOI: 10.1002/14651858.CD004351.pub3

25. Woudstra DM, Chandra S, Hofmeyr GJ, Dowswell T. Corticosteroids for HELLP (hemolysis, elevated liver enzymes, low platelets) syndrome in pregnancy. Cochrane Database Syst Rev. 2010;(9):CD008148. DOI: 10.1002/14651858.CD008148.pub2

26. Juárez-Garcia M, Ortiz-Saavedra P, Gutiérrez-Fernán-Segarra L, Casas-Castañeda J. Patrón y costo del tratamiento antihipertensivo para pacientes ambulatorios en un hospital general. Rev Soc Perú Med Interna. 2008;21(1):17-21. 